\title{
La formación de Trabajadores/as Sociales desde una perspectiva intercultural: aproximaciones desde la práctica pedagógica*
}

\author{
Lilian Sanhueza Díaz ${ }^{* *}$
}

Alicia Rain Rain ${ }^{* * *}$

Luz Huenchucoy Millao ${ }^{* * *}$

RESUMEN

La formación de trabajadores/as sociales en un contexto interétnico e intercultural, como el que presenta la Región de la Araucanía, constituye un enorme desafío, toda vez que requiere pasar del discurso del reconocimiento a la práctica de un reconocimiento y valoración auténticos de la diversidad. Una de las manifestaciones de sufrimiento y malestar social que hoy se hace carne en la Araucanía dice relación con la dificultad histórica que han tenido, tanto el Estado como la sociedad chilena en su conjunto, de reconocer y valorar al pueblo mapuche, su cultura, tradiciones, valores y saberes. En este sentido, el presente trabajo pretende dar cuenta de la incipiente incorporación de saberes propios del pueblo mapuche, en la formación de trabajadores sociales de la Universidad Católica de Temuco (en adelante, UC Temuco).

Palabras clave: Formación profesional, interculturalidad, pueblo mapuche.

\section{A formação de Trabalhadores/as Sociais desde uma Perspectiva Intercultural: Aproximações desde a prática de Pedagógica}

RESUMO

A formação de trabalhadores/as sociais num contexto interétnico e intercultural, como aquele que apresenta a região de Araucanía,

Artículo recibido: 28/01/2014. Artículo aprobado: 22/07/2014. Versión final: 18/08/2014.

** Chilena. Trabajadora Social, Magíster en Intervención Social, Mención Familias y Resolución de Conflictos de la Universidad Autónoma de Chile. Docente Departamento de Trabajo Social de la Universidad Católica de Temuco, Chile. Mail: Isanhueza@uct.cl

*** Chilena. Trabajadora Social, Magíster en Psicología, Universidad La Frontera. Docente Departamento de Trabajo Social de la Universidad Católica de Temuco, Chile. Correo electrónico: arain@uct.cl

**** Chilena. Trabajadora Social, Licenciada en Trabajo Social, Universidad Católica de Temuco, Chile. Facilitadora Intercultural de Fundación La Frontera. Correo electrónico: millaoluz@gmail.com 
constitui um enorme desafio, cada vez que requer passar do discurso do reconhecimento para a prática de um reconhecimento e valorização autentico da diversidade. Uma das manifestações de sofrimento e mal-estar social que hoje se faz carne na Araucania, diz relação com a histórica dificuldade encontrada, tanto o Estado como a sociedade chilena no seu conjunto, de reconhecer e valorizar o povo mapuche, sua cultura, tradições, valores e conhecimentos. Neste sentido, este trabalho pretende dar conta da inicial incorporação dos conhecimentos próprios do povo mapuche na formação de trabalhadores sociais da Faculdade Católica de Temuco.

Palavras chave: formação profissional, interculturalidade, povo mapuche.

\title{
Social workers training from an intercultural perspective: Approaches from teaching practice
}

\author{
ABSTRACT
}

The training of social workers in an interethnic and intercultural context such as the Araucania region is a major challenge, since it needs to move from a theoretical recognition to a practical one with a genuine recognition and appreciation for diversity. One of the manifestations of suffering and social discomfort today in Araucania is related to the historical difficulties faced by both the State and the Chilean society as a whole, to recognize and value the Mapuche people, their culture, traditions, values and knowledge. In this sense, the present study seeks to explain the emerging incorporation of the Mapuche people knowledge in training social workers in Catholic University of Temuco.

Keywords: Vocational training, multiculturalism, Mapuche people.

\section{Antecedentes}

En diversos países de América Latina se han realizado intentos para avanzar en prácticas de mayor inclusión e interculturalidad en la educación superior, por la vía de constituir universidades interculturales especialmente orientadas a las poblaciones indígenas, ubicadas en regiones densamente indígenas, o bien abriendo las puertas de las instituciones universitarias convencionales a poblaciones culturalmente diversas (Schmelkes, 2012; Tubino, 2012). Lo anterior, producto de un proceso reflexivo respecto de la necesidad de formar cuadros profesionales indígenas que aporten al desarrollo de sus comunidades desde su propia visión y aspiraciones, minimizando el proceso de aculturación que una educación homogeneizante provoca (Prada y López, 2012; Schmelkes, 2012). 
En nuestro país, se han observado algunos avances a nivel de la enseñanza básica y media, particularmente a través de la Educación Intercultural Bilingüe y en algunas universidades en la formación de docentes en carreras de pedagogía ${ }^{\prime}$; sin embargo, son escasas las experiencias de incorporación de saberes mapuche en la formación de otros profesionales de las ciencias sociales en general y de trabajo social en particular. Aun cuando se han ido introduciendo en los currículos contenidos vinculados con los saberes y tradiciones culturales desde hace mucho tiempo, desde las ciencias sociales "el tratamiento dado a estos temas es discutible y hoy (...) los intelectuales indígenas, afirman que se ha reconstruido y reproducido en las aulas universitarias una versión oficial de la historia; destacando que la percepción hegemónica se ha constituido en el filtro -también ideológico y político- con el cual se ha analizado todo lo subalterno" (Prada y López, 2012:38). Es decir, se ha construido una visión académica hegemónica, a partir de la cual las ciencias sociales estudian e interpretan a "los otros" desde sus particulares criterios de investigación y construcción de conocimiento, perpetuando la subalternidad de los pueblos indígenas en general y del pueblo mapuche en particular. Acá lo relevante, según Ansión (2012), es trabajar la "relación entre las culturas", ya que ha existido un ocultamiento de los saberes indígenas, dando cuenta de un racismo que persiste en forma "solapada" en las academias.

Las formas de colonialismo se han expresado en la estructura política, económica y cultural, pero esto se ha trasladado al mundo cotidiano de las relaciones humanas. Esto para el caso del pueblo mapuche, según Caniuqueo (2009), sería el "disciplinamiento social de los Mapuche", que ha generado un trauma social para quienes pertenecen a este pueblo y que se traspasa a diversas dimensiones de la vida de las personas.

No es casual que en las regiones donde existen altos porcentajes de población indígena y afro-descendientes, se encuentren los índices de mayor inequidad social y económica (Hopenhayn y Bello, 2000), como es la Región de la Araucanía en Chile. La última Encuesta de Caracterización Socioeconómica (CASEN; 2011), muestra que la Región de la Araucanía presenta el índice de pobreza

Un ejemplo de ello lo constituye la carrera de Educación Básica Intercultural impartida por la Universidad Católica de Temuco, Chile. 
más alto del país con un 22,9\%, y una importante presencia de población mapuche que equivale al 33,6 \% del total de habitantes de la región (Educar Chile, 2014). Estos antecedentes nos revelan la necesidad de pensar la educación bajo un "paradigma humanista" como lo expone Tubino (2012), en donde se visualicen las desigualdades, las inequidades y las posibilidades de diálogo.

El fenómeno de la masificación de la educación superior ha abierto la oportunidad a miles de jóvenes para iniciar estudios superiores. Particularmente para el caso de Trabajo Social en la Universidad Católica de Temuco, muchos estudiantes que ingresan a esta carrera cuentan con trayectorias vitales que muestran condiciones de vulnerabilidad, pero a su vez recursos y potencialidades que les han permitido afrontar de modo resiliente las dificultades asociadas a provenir de establecimientos educacionales con bajos resultados SIMCE, familias donde ellos constituyen la primera generación en llegar a la universidad y, en más del 70\% de los matriculados, provenir de los dos primeros quintiles socioeconómicos (Informe CRA, 2012, 2013).

De este modo, optar por una docencia pertinente al contexto sociocultural y centrada en el estudiante implica varios desafíos a la formación profesional en Trabajo Social para la Araucanía, al comprender la actividad académica como una práctica que requiere una reflexión permanente y la renovación de metodologías y contenidos que respondan a las necesidades del contexto en que se desarrolla el proceso formativo. Estas acciones darían paso a pensar la educación como un espacio para la "reivindicación" y consecutivo "reconocimiento", según Ansión (2012).

Una apuesta concreta es que la academia pueda abrirse a una "pluri-versalidad epistemológica" que puede generarse más allá de los cánones establecidos por el cientificismo occidental, conociendo lo que las comunidades indígenas y afro-descendientes tienen por entregar (Wlash, 2007).

\section{La formación de trabajadores sociales en contexto mapuche}

La construcción de una sociedad intercultural es un proceso social complejo y determinado por las variables sociohistóricas de cada país, cuyas bases requieren un cuerpo jurídico y normativo 
basado en el reconocimiento constitucional de los pueblos indígenas y del derecho propio indígena (la costumbre), así como de políticas públicas inclusivas y respetuosas de la diversidad cultural; sin embargo, la transformación efectiva de la sociedad requiere también de procesos educativos que incorporen desde la educación pre escolar hasta la educación superior, los conocimientos y saberes de nuestros pueblos indígenas. De este modo, aunque "la educación no es sino un componente más que contribuye a la configuración y transformación de estas sociedades" (Prada y López, 2012:36), es de gran relevancia en este proceso.

El trabajo social, como disciplina que se ha abocado a trabajar con aquellos sectores históricamente subordinados, comparte en alguna medida esta condición en el concierto de las ciencias sociales; sin embargo, su "modo" fenomenológico y hermenéutico de acercarse al mundo de "los otros", le confiere una ventaja comparativa en términos de su ontología disciplinar, puesto que se encuentra más cercana a las propias vivencias de los sujetos y cuenta con una trayectoria de escucha y acompañamiento que constituyen prácticas necesarias para avanzar en el camino de la interculturalidad.

Para el trabajo social "el quehacer profesional se hace en el encuentro con el otro, en procesos intersubjetivos, aboga por la propia persona de los que están comprometidos en esta situación social específica" (Aguayo, 2006:122). De este modo, la formación de trabajadores sociales en la Araucanía nos enfrenta al desafío de incluir saberes propios del pueblo mapuche con una doble finalidad: por una parte para generar estrategias de acción profesional con pertinencia cultural y por otra, favorecer la identidad cultural de los estudiantes mapuche de trabajo social en la UC Temuco.

Respecto de la primera finalidad, se observa que al incorporar conocimiento "desde" el mundo mapuche a los estudiantes se contribuye a disminuir el proceso de aculturación propio de una formación profesional basada en un currículo homogeneizante donde "se consagran como oficiales y, por ende, como nacionales e incluso universales sólo los valores y conocimientos que forman parte del acervo cultural e ideológico del sector hegemónico; y desde esta posición se excluye todo saber no-oficial "(Prada y López, 2012:33). 
En la práctica cotidiana de la profesión, observamos que la relación de los profesionales del trabajo social con los actores sociales "está en el encuentro de los límites de la subjetivización, es su propia subjetividad la que está puesta en cuestión. Es su confrontación con los límites del derecho, es su propia relación a la norma la que es interrogada" (Autes; 1999, citado en Aguayo, 2006). Así, la formación profesional en un contexto de relaciones interétnicas e interculturales desafía a los estudiantes mapuche y no mapuche, a los docentes mapuche y no mapuche y a los planes de estudio a incorporar elementos que permitan a los estudiantes este cuestionamiento respecto las concepciones normativas acerca de temas fundamentales para el trabajo social, como lo son la concepción de persona, familias y comunidades. Este tipo de apuesta para Tubino (2012) sería la de "interculturalizar" una universidad desde acciones que favorezcan el "reconocimiento" de los indígenas y sus saberes.

Cuando compartimos como seres humanos una misma racionalidad, una misma forma de ver el mundo y entender la vida, el ejercicio profesional se basta a sí mismo en tanto cuenta con un cuerpo de conocimientos, técnicas y normas éticas que le dan legitimidad en un contexto social y cultural determinado. Sin embargo, en contextos de diversidad cultural caracterizados por procesos históricos de negación y subordinación, como es el caso de la relación entre el pueblo mapuche y la sociedad chilena, se hace imprescindible repensar la formación de los profesionales de la acción social, particularmente de los trabajadores(as) sociales, dadas las implicancias éticas y humanas que conlleva el ejercicio de la profesión con sujetos que constituyen un "otro" diferente. Se trata de pensar una "nueva forma de universalismo" que permita ver de modo crítico el proceso de construcción entre saberes distintos (Ansión, 2012).

La incorporación de saberes mapuche en la formación de trabajadores sociales de la UC Temuco ha ido evolucionando, de modo que desde el año 2008 se ha intencionado que estos saberes no sean transmitidos por profesionales de las ciencias sociales que estudian "lo mapuche", sino por los propios mapuche, a través de sus autoridades ngempin, kimche, chachay, ñaña, entre otros, en la línea de la descolonización del saber. De esta manera se busca trascender el "control disciplinario" que plantea Foucault (1973, 1978), en la medida que ya no son los criterios de verdad o false- 
dad de las ciencias sociales los que legitiman el saber indígena, sino sus propios actores convocados a compartir su saber. En este sentido, nos hacemos parte de la convocatoria de López (2012) "a pensar", acción que "a diferencia de conocer, que consiste en un salir del sujeto a la búsqueda/construcción de un objeto (...), es fruto de la escucha atenta de lo que nos interpela" (López, 2012:25).

Esta estrategia de incorporación de saberes mapuche constituye un compromiso ético y político frente a la necesidad de develar y transformar las relaciones de discriminación hacia los pueblos indígenas, particularmente hacia el pueblo mapuche en la Región de la Araucanía (Merino, 2009). A este respecto, compartimos los planteamientos de Tubino respecto a que "el problema de la discriminación étnica y cultural no es un problema exclusivo de los discriminados. La discriminación es una relación de a dos. Atacar la discriminación en sus causas implica, por lo tanto, un trabajo intenso y sistemático de educación intercultural no sólo con los sectores discriminados sino también con los sectores hegemónicos y discriminadores de la sociedad. "Interculturalidad sí, pero para todos" es pues una necesidad impostergable si queremos recomponer el tejido social y cultural de nuestras sociedades estructuralmente segmentadas. Es la condición de posibilidad de la refundación del pacto social, el principio rector de los Estados multiculturales que nuestras sociedades requieren" (2004:156). Así, a pesar de que nuestro país no se ha definido constitucionalmente como pluricultural, es pues esta una condición de facto que no puede ser negada ni invisibilizada por quienes son responsables de la formación profesional.

\section{Bases para una acción pedagógica situada y aplicada}

En nuestro país podemos observar diariamente la negación del otro como práctica recurrente y, en este sentido, se hace comprensible que cotidianamente se observe entre los niños, jóvenes y adultos lo que Maturana y Vignolo (2001) han venido a denominar la exageración de las exigencias que conducen a la destrucción del otro. En este contexto, se incorpora en la temprana socialización de los niños la conformación de ideas preconcebidas sobre aquellos que son considerados ajenos; el punto no sería 
tan relevante si no fuese porque dichas preconcepciones son persistentemente negativas y conducen a la generación de prejuicios y estereotipos sobre otros, pertenecientes a culturas, religiones o ideologías diferentes.

Los contextos educativos históricamente se han constituido en un medio para el despliegue de estrategias de dominación y ejercicio agresivo del poder de la sociedad hegemónica, ya que sus prácticas históricas han sido las de negar que los saberes populares e indígenas son válidos (Wlash, 2007). En este sentido, nos enfrentamos hoy a un nuevo desafío para la universidad "que le corresponde (...) precisamente por ser pieza clave del patrón del poder y del saber imperantes, someter a análisis el saber que produce y transmite, para des-ocultar su relación con el poder y traer a la presencia la violencia epistémica y simbólica de la que ese saber es portador" (López, 2012:23).

Freire (1970) nos convoca a mirar la educación como un espacio, como una oportunidad en la que se pueda dar paso a la experiencia como un saber válido y así mismo como proceso de reflexión y análisis permanente, en donde la conciencia es el espacio y el camino para la liberación, por tanto para la autoconfiguración responsable.

El sistema educativo ha promovido una relación asimétrica entre docente y estudiante, en donde el conocimiento reconocido y valorado ha sido el del profesor, como si los estudiantes llegasen sin ningún tipo de experiencia y de saber a la educación formal. En este sentido Maturana y Vignolo (2001) brindan especial relevancia al profesor, ya no como consideración única del conocimiento como contenido, sino por la capacidad que éste tenga de poder abrir o cerrar espacios para los aprendizajes en la educación. Esto es precisamente lo que nos convoca en esta búsqueda de generar espacios de diálogo y encuentro de nuestra comunidad estudiantil, abriendo las aulas de clases para que sean ellos quienes compartan su experiencia y conocimiento, el cual se encuentra situado, es local y acorde a nuestra realidad como región pluricultural.

Vemos que este tipo de prácticas no es más que una oposición a una cultura tejida de una trama de dominación, por lo que como establece Freire (1970), sería una forma de aproximación a un continuo retornar reflexivo que permite por tanto, acercar- 
se a la liberación. En cuanto a estos postulados, la alfabetización implicaría no sólo la entrega de conocimientos técnicos y metodológicos, sino que involucraría un ejercicio afectivo y de conciencia social que ya no se ejercita sólo hacia lo externo, sino que se operacionaliza y se aplica habitualmente en el proceso de aprender y desaprender cotidiano.

En palabras de Freire, el alfabetizando gana distancia para ver su experiencia, "ad - mira", en ese mismo instante comienza a decodificar, siendo esto un análisis y reconstitución de la situación vivida que es en sí misma, una posibilidad concreta de pasar más allá, se va haciendo crítica, en tanto que la observación de su propia realidad ya no se haría desde una visión rígida, sino más bien se generaría una circularidad reflexiva, que compromete una descomposición permanente de lo vivido.

Lo anterior se expone como experiencia de la acción que desde la Carrera de Trabajo Social se ha intentado llevar a cabo, en cuanto a que al no ser los docentes quienes lideren el dar a conocer principales valores, principios, concepciones de familia y estrategias de crianza mapuche a los estudiantes, se ha podido observar que el diálogo y la forma de interacción es más fluida y mayormente significativa. Con estas acciones se ha pretendido que los estudiantes reconozcan la presencia y el valor que revisten estos saberes, por tanto que aprendan que la capacidad de escuchar es una posibilidad de reencontrarse con los otros y en los otros compañeros, en lo que Freire denomina "su círculo de cultura". Estas acciones son así mismo una instancia favorable para que el docente pueda replantearse críticamente.

\section{Pasos hacia la incorporación de saberes Mapuche en la formación de Trabajadores(as) Sociales}

La carrera de Trabajo Social se imparte en la UC Temuco desde el año 1992, a través de un diseño curricular que se va revisando y actualizando, pero que en términos de estrategia pedagógica y contenido mantiene su esencia hasta el año 2008.

En el marco de la implementación del Nuevo Modelo Educativo de la UC Temuco, el equipo docente resuelve en el año 2006, ini- 
ciar un proceso de renovación curricular que responde a la necesidad de actualizar los contenidos y prácticas docentes, comenzando por la reflexión y análisis de la disciplina y la profesión. Las interrogantes sobre la adecuación y pertinencia formativa de la profesión en los nuevos escenarios sociales y laborales, la discusión acerca del rol ético-político de los/as trabajadores sociales y los fundamentos epistemológicos de una profesión históricamente subordinada, posibilitaron un proceso de rediseño curricular centrado en el aprendizaje significativo del estudiante, la formación por competencias ${ }^{2}$ y la articulación de los aprendizajes en torno a una Identidad de la Carrera o Perfil de Egreso, al cual todas las competencias y contenidos de las asignaturas deben contribuir.

Luego de un proceso eminentemente reflexivo y crítico, el equipo académico de la Escuela de Trabajo Social de la UC Temuco define la Identidad de la Carrera de la siguiente manera:

"La Carrera de Trabajo Social de la Universidad Católica de Temuco forma profesionales de excelencia, desde una orientación cristiana, preparados en el trabajo con personas en los ámbitos familiar, comunitario y organizacional, con el fin de contribuir al mejoramiento de su calidad de vida y al desarrollo de la región y de la sociedad en general. Para ello realiza acciones orientadas a la transformación social y a la producción de conocimiento teórico-práctico, articulando cosmovisiones, saberes científicos y locales, valorando la diversidad étnica y sociocultural, a través de una relación ético-dialógica con los actores sociales" (Documento Perfil de Egreso Carrera de Trabajo Social; 2008:2).

Esta identidad de carrera, que en el itinerario formativo se desarrolla a través de competencias específicas, surge en coherencia con el compromiso asumido por la UC Temuco al establecer como una de las competencias genéricas identitarias ${ }^{3}$ en la for-

Para el Modelo Educativo de la UC Temuco se entiende por Competencia: “Un saber actuar movilizando recursos propios y ajenos para resolver problemas reales de manera efectiva y éticamente responsable, con creatividad e innovación. Los recursos se refieren de manera especial a los distintos saberes (ser, saber y saber hacer) que de manera integrada se transforman en dispositivos que serán utilizados por la persona competente" (Modelo Educativo UC Temuco:2009)

Competencias Identitarias son aquellas competencias genéricas declaradas por la UC Temuco como sello de su formación profesional y, por tanto, deben ser trabajadas en todas las carreras hasta su máximo grado de desarrollo, esto es hasta alcanzar el Nivel 3. 
mación de todos los estudiantes de la universidad el Respeto y

Valoración de la Diversidad. Lo anterior aparece en consonancia con lo planteado por Ansión en virtud de que "en ocasiones es preciso un trabajo intracultural previo al trabajo intercultural" (2012: 90), siendo este un primer paso para avanzar en esta vía.

Buscar espacios de encuentro con el otro distinto, es una inquietud constante tanto para los(as) estudiantes mapuche, como para los(as) profesionales mapuche, a partir de las características particulares que adquiere la filosofía de vida y el mongen (vida) en el mundo mapuche. Incorporar el mapuche kvmvn en las aulas es una inquietud transmitida por los antiguos abuelos "chew rume amulmy ngoymalayamy tamy mapuche kvmvn, tamy Rakizuam, kom tamy pu che, femlymy ta ngoymayaymy inche ka eymy", en cualquier lugar donde andes nunca olvides el conocimiento mapuche, nuestro pensamiento, tu gente, porque si lo haces te estás olvidando de tu gente, de mí, y a la vez de ti misma, o sea no existes. (Ngenpin Domingo Millao ${ }^{4}$ ).

El currículum de formación en Trabajo Social de la UC Temuco incluye un Área de Formación General y un Área de Formación Específica. La primera, incluye los cursos interdisciplinarios y de sello institucional que la universidad dicta para todas las carreras y que ponen un fuerte énfasis en las competencias genéricas identitarias de la UC Temuco: Actuación ética y respeto a la diversidad; y en el Área de Formación Específica se concentra el 80\% de los créditos y en ella se distinguen los siguientes ámbitos: Ciencias sociales y humanas, Trabajo social e investigación social.

Han sido los cursos del ámbito del Trabajo Social en los cuales se han iniciado los procesos de incorporación de saberes mapuche de manera sistemática desde el 2008 a la fecha, particularmente en los cursos de Aproximación a la realidad social I (TSO 1110), Trabajo Social y Familia II (TSO 1143) y Trabajo Social, Pueblo Mapuche y Praxis Intercultural (TSO 1137).

En el curso de Aproximación a la realidad social I, se inicia el año 2009 incorporando como resultado de aprendizaje: El (la) estudiante distingue los distintos saberes que interpretan la realidad

Consejo transmitido por el bisabuelo a la entonces estudiante mapuche Luz Huenchucoy Millao, actual trabajadora social y coautora del presente artículo. 
social -científico, indígena y popular- a través de la revisión de un artículo científico, la presentación de Ngenpin y la entrevista a un sabio popular. A partir de lo anterior, cada año se ha contado con la participación del ngenpin Armando Marileo, quien ha presentado aspectos de la filosofía mapuche logrando una favorable acogida y una alta valoración de parte de los estudiantes.

En la asignatura Trabajo Social y Familia II, el año 2008, se invita al Kimche Manuel Manquepi ${ }^{5}$ a compartir los conocimientos vivenciales acerca de los procesos de socialización y formación de personas mapuche, estrategias propias del pueblo mapuche para asegurar el traspaso de conocimiento, el canto, la poesía, los epeu ${ }^{6}$, los nütram ${ }^{7}$, entre otros.

En este mismo curso, durante el año 2009, 2010, 2011 y 2012, se cuenta con la participación de la ñaña Camila Llanquinao Trabol ${ }^{8}$, quien da a conocer a los estudiantes diversas prácticas y conocimientos de las formas de crianza, valores y estructuras familiares del Pueblo Mapuche. La profesional transmite la importancia de la realización del meli folil kupan ${ }^{9}$, como instrumento pertinente para ser aplicado en contexto mapuche, en donde se desprenden dos conceptos importantes, el tuwvn ${ }^{10} \mathrm{y}_{\text {kvpalme }}{ }^{11}$, ayudando a comprender la situación familiar que involucra un espacio de encuentro con su reñmawen ${ }^{12}$.

La profesional, en estas oportunidades, incentiva a los estudiantes a profundizar sus conocimientos sobre esta cultura y su len-

5 Fue docente de la Facultad de Educación de la Universidad Católica de Temuco, coordinador Regional de la Academia de la Lengua Mapuche y co-autor de publicaciones destinadas a relevar el conocimiento cultural.

6 Enseñanzas por medio de relatos.

7 Consejos entregados a los niños y jóvenes a cargo de ancianos, abuelos y sabios mapuche.

8 Trabajadora Social y Magíster (c) en Interculturalidad de la UC Temuco, actual Jefe Técnico de Libertad Asistida Especial de Fundación Tierra de Esperanza de Temuco. Además, es Coordinadora Regional de la Academia de la lengua Mapuche y coautora en libros y artículos vinculados a la cultura mapuche y su lengua.

9 Instrumento que surge en el contexto de educación básica intercultural y que sirve para sintetizar la estructura e historia familiar, relevando el Kvpalme (linaje) y el Tüwvn (historia del territorio al que corresponde la familia).

10 Importancia de la historia del territorio para definir características de personas que pertenecen al mismo y configuración de su identidad (su AZ).

11 Historia familiar de los ancestros en cuanto a cargos tradicionales ceremoniales u otros, linaje, tipos de familia, valoración de la comunidad, entre otros.

12 Principal valor que se enseña en la cultura mapuche a los niños y niñas, siendo relevante valorar los vínculos afectivos con las familias, el respeto y cercanía. 
gua, lo que favorece la creación de un taller de mapuzungun y filosofía mapuche para estudiantes de la carrera de Trabajo Social; el cual es desarrollado por la entonces egresada de trabajo social Luz Huenchucoy Millao por iniciativa de los mismos estudiantes durante el año 2010. A partir de esta experiencia, desde la Carrera de Trabajo Social, se aprueba la generación de un optativo de profundización denominado "Mundo Mapuche y Vida Familiar Mapuche", el cual es diseñado durante el primer semestre de 2011 para ser impartido a estudiantes de cuarto y quinto año de la carrera el segundo semestre de este año.

Finalmente, el curso de Trabajo Social, Pueblo Mapuche y Praxis Intercultural, fue pensado desde el inicio de la renovación curricular como un espacio de diálogo y encuentro intercultural. Este curso se ha dictado de manera colegiada por una docente mapuche hablante de mapuzungun y una docente no mapuche con formación en filosofía, las cuales buscan generar un diálogo filosófico occidental-indígena.

Todas estas innovaciones han sido altamente valoradas por los estudiantes, sin embargo se observa la necesidad de avanzar en la incorporación de saberes y actores mapuche en otras asignaturas del ámbito del Trabajo Social tales como aquellas vinculadas al trabajo comunitario y en el ámbito de la investigación social.

\section{Nuestras aspiraciones como comunidad académica y social}

Consideramos que la educación y formación profesional de futuros trabajadores sociales requiere de un tratamiento exhaustivo no sólo de la capacidad técnica, sino también de potenciar el desarrollo de competencias que permitan un acercamiento comprensivo a las realidades sociales, familiares y humanas de las personas con las cuales les corresponda interaccionar. Compartimos la necesidad de ir construyendo un proyecto académico que permita recuperar el saber local (Wlash, 2007), el conocimiento propio del pueblo mapuche y de ese modo contribuir a un proceso de más largo aliento de descolonización del conocimiento y de la academia o, en palabras de Ansión (2012), desarrollar "relaciones de interaprendizaje", por medio del empoderamiento de estudiantes pertenecientes a sociedades subalternas, como las 
indígenas (Prada y López, 2012) y a un reconocimiento de estos saberes en los estudiantes no mapuche.

Actualmente nos encontramos "mirando" y "pensando" el saber mapuche que emerge desde la alteridad, pero aún nos falta avanzar en el camino de "pensar" a nuestros propios estudiantes mapuche, reconocer sus saberes y articular una formación docente que releve este conocimiento y que diversifique estrategias pedagógicas con pertinencia cultural. La academia no puede continuar en el afán "cientificista occidental" y negar los saberes indígenas (Wlash, 2007).

Un desafío pendiente lo constituye el reconocer que la función docente debe avanzar "hacia la construcción de conocimientos en el contexto donde trabaja y con los estudiantes con los que interactúa. La educación contemporánea exige un docente investigador de las culturas de sus estudiantes para poder aprovechar sus conocimientos previos y así crear una dinámica pedagógica que no produzca saberes subalternos" (Prada y López, 2012:41).

Como docentes en trabajo social consideramos necesario repensar la formación a la luz de las contingencias sociales, culturales y políticas actuales, particularmente atendiendo al perfil de egreso académico profesional que define al profesional como un actor capaz de aportar a la transformación social de los diversos malestares sociales presentes en nuestra sociedad. En este sentido, la incorporación de actores y saberes propios del mundo mapuche constituye un insumo para los necesarios procesos de reflexión y análisis de la diversidad cultural. Se hace necesario avanzar en la apertura de las fronteras académicas que permitan al estudiante estar en contacto con las dinámicas de las sociedades con las cuales trabajará de forma conjunta en su futuro ejercicio profesional, de manera tal que dicho hábito se forje a tiempo y evitemos realizar acciones descontextualizadas de la realidad.

En relación a la investigación, aparece la necesidad de un "desplazamiento epistemológico" que nos convoque a explorar metodologías de investigación participativas, basadas en una episteme de corte fenomenológico y hermenéutico; e inclusive ponernos en disposición de abandonar nuestros propios métodos para incluir formas alternativas de construcción del conocimiento propias de los pueblos indígenas, métodos basados por ejemplo 
en la oralidad más que en la tradición escrita de Occidente. En este sentido, hemos avanzado en la generación de trabajos de título desarrollados por estudiantes mapuche acerca de temáticas de relevancia para el trabajo social y que rescatan la voz de los propios actores del pueblo mapuche desde una perspectiva hermenéutica.

Requerimos continuar en la búsqueda de espacios y formas de diálogo que contengan de forma lo suficientemente comprensiva las diferencias y particularidades de nosotros mismos y de quienes nos rodean, siendo ello finalmente un caminar hacia relaciones más humanas, más éticas y de mayor respeto y valoración. Esto es lo que Bateson (1991) denomina cambios epistemológicos o de distintas epistemologías; se refiere a diversos modos de vida, distintos espacios psíquicos conscientes e inconscientes. En palabras de Maturana y Vignolo (2001), en el dominio de lo humano el entendimiento y la acción van juntos y este es un desafío permanente para el trabajo social como disciplina y para quienes formamos a profesionales del trabajo social: avanzar en un proceso recursivo que genere conocimiento desde y para la práctica -tanto profesional como pedagógica- en un diálogo genuino con la alteridad.

\section{Bibliografía}

Aguayo, C. (2006). Las Profesiones Modernas. Dilemas del Conocimiento y del Poder. Santiago: Ediciones Universidad Tecnológica Metropolitana.

Ansión, J. (2012). Universidad e Interculturalidad en el Perú, en Tubino, F. y Mansilla, K. (editores), Universidad e Interculturalidad: desafíos para América Latina, pp. 87-106. Lima, Perú: Pontificia Universidad Católica del Perú.

Bateson, G. (1991). Pasos hacia una ecología de la mente. Buenos Aires: Carlos Lohlé - Planeta.

CASEN (2011). Encuesta de caracterización socioeconómica. Ministerio de Desarrollo Social. Chile.

Caniuqueo, S. (2009), Particularidades en la instauración del colonialismo chileno en el Gulu Mapu, 1884-1950. Subordinación, alianzas y complicidades. En Martínez, C. y Estrada, M. (coord.), Las disputas por la etnicidad en América Latina: movilizaciones indí- 
genas en Chiapas y Araucanía, pp. 191-212. Santiago de Chile: Editorial Catalonia.

Educar Chile: http://www.educarchile.cl/ech/pro/app/detalle? ID $=130271$

Foucault, M. (1973). El orden del discurso. Barcelona: Tusquets editores.

Foucault, M. (1978). Vigilar y Castigar. México: Editorial Siglo XXI.

Freire, P. (1967). La educación como práctica de la libertad. México: Ed. Siglo XXI.

Freire, P. (1970). Pedagogía del Oprimido (2a Edición). Buenos Aires, Argentina: Editorial Tierra Nueva y Siglo XXI.

Hopenhayn, M. y Bello, A. (2000). Discriminación étnico-racial y xenofobia en América Latina y el Caribe. División de Desarrollo Social, Serie Políticas Sociales, N 47. Santiago de Chile: CEPAL.

Informe CRA (2012-2013). Informe Carrera de Trabajo Social. Centro de Recursos para el Aprendizaje CRA. Temuco, Chile: Universidad Católica de Temuco.

López, J. (2002). Universalidad e interculturalidad. En Tubino, F. y Mansilla, K. (editores). Universidad e Interculturalidad: desafíos para América Latina, pp.13-28. Lima, Perú: Pontificia Universidad Católica del Perú.

Vignolo, C., Maturana, H. (2001). Conversando sobre Educación, Revista Perspectivas en Política, Economía y Gestión, Vol $4-\mathrm{N}^{\circ} 2$, pp. 249- 266. Santiago de Chile: Departamento de Ingeniería Industrial, Universidad de Chile.

Merino, M. E. (2009). Análisis del discurso en las relaciones interétnicas: conflictos y consecuencias. En Fuentes, L. (Coord.). La universidad y la atención a la diversidad cultural: de la discriminación a la inclusión. Congreso Internacional, Sede Regional Villarrica, Chile: Pontificia Universidad Católica de Chile.

Prada, F. y López, L. (2012). Educación superior y descentramiento epistemológico. En Tubino, F. y Mansilla, K. (editores). Universidad e Interculturalidad: desafíos para América Latina, pp. 29-54. Lima, Perú: Pontificia Universidad Católica del Perú.

Schmelkes, S. (2012). Multiculturalismo, educación intercultural y universidades. En Tubino, F. y Mansilla, K. (editores). Universidad e Interculturalidad: desafíos para 
América Latina, pp. 55-85. Lima, Perú: Pontificia Universidad Católica del Perú.

Tubino, F. (2004). Del Interculturalismo Funcional al Interculturalismo Crítico. En Garbarini, C. E Samaniego, M Rostros y Fronteras de la Identidad. Temuco, Chile: Ediciones Universidad Católica de Temuco.

Tubino, F. (2012). Formación universitaria para el desarrollo humano abierto a la diversidad. En Tubino, F. y Mansilla, K. (editores), Universidad e Interculturalidad: desafíos para América Latina, pp.107-124. Lima, Perú: Pontificia Universidad Católica del Perú.

Universidad Católica de Temuco (2007). Modelo Educativo UC Temuco. Principios y lineamientos.

Universidad Católica de Temuco (2008). Perfil de Egreso Carrera de Trabajo Social. Documento de Trabajo. Escuela de Trabajo Social. Material no publicado.

Wlash, C. (2007). ¿Son posibles unas ciencias sociales/culturales otras? Reflexiones en torno a las epistemologías decoloniales. Revista Nómadas, $\mathrm{N}^{\circ} 26$, pp. 102-103. Colombia: Universidad Central. 
